\title{
3D YSO accretion shock simulations: a study of the magnetic, chromospheric and stochastic flow effects
}

\author{
T. Matsakos ${ }^{1,2,3}$, J.-P. Chièze ${ }^{2}$, C. Stehlé ${ }^{3}$, M. González ${ }^{4}$, L. Ibgui ${ }^{3}$,

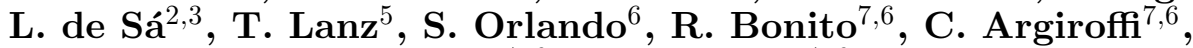 \\ F. Reale ${ }^{7,6}$ and G. Peres ${ }^{7,6}$ \\ ${ }^{1}$ CEA, IRAMIS, Service Photons, Atomes et Molécules, 91191 Gif-sur-Yvette, France \\ email: titos.matsakos@cea.fr \\ ${ }^{2}$ Laboratoire AIM, CEA/DSM - CNRS - Université Paris Diderot, IRFU/Service \\ d'Astrophysique, CEA Saclay, Orme des Merisiers, 91191 Gif-sur-Yvette, France \\ ${ }^{3}$ LERMA, Observatoire de Paris, Université Pierre et Marie Curie and CNRS, 5 Place J. \\ Janssen, 92195 Meudon, France \\ ${ }^{4}$ Université Paris Diderot, Sorbonne Paris Cité, AIM, UMR 7158, CEA, CNRS, 91191 \\ Gif-sur-Yvette, France \\ ${ }^{5}$ Laboratoire Lagrange, Université de Nice-Sophia Antipolis, CNRS, Observatoire de la Côte \\ d'Azur, 06304 Nice cedex 4, France \\ ${ }^{6}$ INAF - Osservatorio Astronomico di Palermo, Piazza del Parlamento 1, 90134 Palermo, Italy \\ ${ }^{7}$ Dipartimento di Fisica e Chimica, Università degli Studi di Palermo, Piazza del Parlamento \\ 1, 90134 Palermo, Italy
}

\begin{abstract}
The structure and dynamics of young stellar object (YSO) accretion shocks depend strongly on the local magnetic field strength and configuration, as well as on the radiative transfer effects responsible for the energy losses. We present the first 3D YSO shock simulations of the interior of the stream, assuming a uniform background magnetic field, a clumpy infalling gas, and an acoustic energy flux flowing at the base of the chromosphere. We study the dynamical evolution and the post-shock structure as a function of the plasma-beta (thermal pressure over magnetic pressure). We find that a strong magnetic field ( hundreds of Gauss) leads to the formation of fibrils in the shocked gas due to the plasma confinement within flux tubes. The corresponding emission is smooth and fully distinguishable from the case of a weak magnetic field ( tenths of Gauss) where the hot slab demonstrates chaotic motion and oscillates periodically.
\end{abstract}

Keywords. accretion, magnetohydrodynamics (MHD), radiative transfer, shock waves, instabilities

\section{Introduction}

The accretion process in young stars occurs by plasma streams that originate from the inner part of the surrounding disk and flow along the field lines of the magnetosphere before hitting the stellar surface. The plasma impacts onto the chromosphere with freefall velocities resulting in the formation of strong shocks. The temperature that develops in the post-shock region is on the order of a few million Kelvin, which leads to the emission of soft X-ray photons. Recent observations of several protostars seem to have detected such radiation (e.g. Argiroffi et al. 2007). 

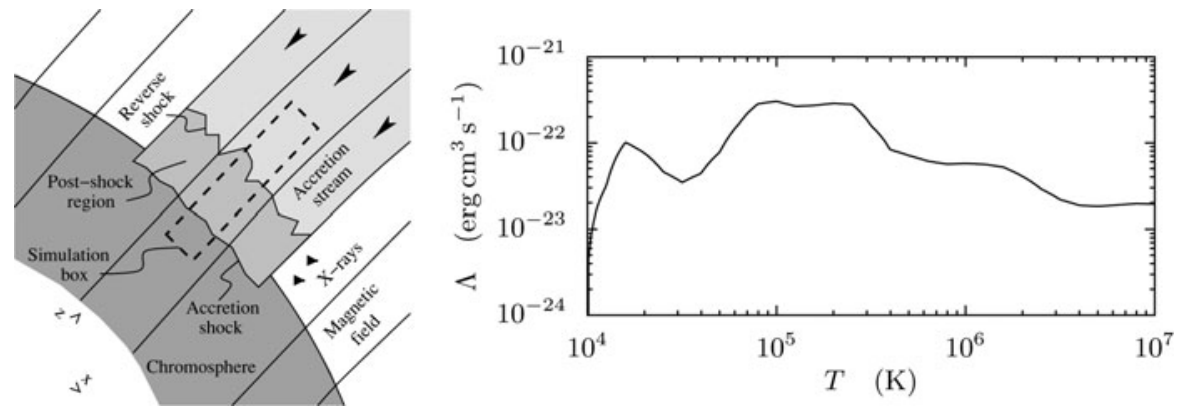

Figure 1. Left panel: a simplified sketch of an accretion shock for a strong, uniform magnetic field. The computational domain is indicated with dashed lines. Right panel: the prescribed cooling function Orlando et al. 2010, Matsakos et al. 2013.

Numerical studies have explored the dynamics of accretion shocks in 1D and 2D (e.g. Sacco et al. 2008, 2010; Koldoba et al. 2008; Orlando et al. 2010, 2013; Matsakos et al. 2013). To highlight the evolution of the system, consider the simplified configuration depicted in the left panel of Fig. 1 in 1D, namely focus on the interior of the stream and assume a uniform magnetic field. After the impact, a reverse shock travels along the stream as material accumulates building a hot slab. The optically thin radiation losses that are often adopted to describe the post-shock region, see right panel of Fig. 1, trigger cooling instabilities. Specifically, as the plasma cools, the infalling gas can no longer be supported due to the large pressure drop. As a result, the reverse shock collapses and a new hot slab starts to build up again (Sacco et al. 2008; Koldoba et al. 2008). This formation-collapse cycle of the post-shock region suggests a quasi-periodic behavior for the observable X-ray emission, with a frequency of $10^{-3}-10^{-1} \mathrm{~Hz}$. However, observations do not yet find evidence of periodicity (Drake et al. 2009; Günther et al. 2010).

The absence of periodic patterns could be due to a variety of physical mechanisms. For instance, radiative transfer effects could complicate the dynamics (de Sá et al. in preparation) or non-uniform magnetic fields could break the 1D symmetry and lead to a complex evolution (Orlando et al. 2010, 2013). Here, we show that even in the simple case of a uniform field and optically thin cooling, the global post-shock oscillations can effectively be suppressed.

We perform 3D magneto-hydrodynamical (MHD) simulations of a vertical element of the interior of the accretion stream (left panel of Fig. 1) using the PLUTO code (Mignone et al. 2007). We introduce realistic perturbations in the system, i.e. clumps in the stream and a chromospheric acoustic flux, and we explore three different models by changing the value of the magnetic field. The computational domain in the horizontal directions spans $x, y=\left[0.0,0.5 \times 10^{-3}\right] R_{\odot}$ and in the vertical $z=\left[0.0,7.7 \times 10^{-2}\right] R_{\odot}$. We use a static grid and a resolution of $32 \times 32 \times 256$ cells.

On the top boundary, as well as the upper part of the domain initially, we prescribe a uniform flow of number density $n_{\text {acc }}=2 \cdot 10^{11} \mathrm{~cm}^{-3}$ and velocity $v_{\text {acc }}=500 \mathrm{~km} \mathrm{~s}^{-1}$. Moreover, we add clumps of random size and location on top of the accretion stream. Their density follows the gaussian distribution with the peak value set at $n_{\mathrm{clm}}=10^{12} \mathrm{~cm}^{-3}$ and standard deviation equal to $10 \%-30 \%$ of the box width $\left(\sim 10^{2}-10^{3} \mathrm{~km}\right)$. On the bottom boundary as well as the lower part of the initial box, we specify an isothermal chromosphere with $T_{\mathrm{chr}}=10000 \mathrm{~K}$ that is in equilibrium with the imposed constant gravity. The protostar is assumed of solar mass and radius. For more information on the setup, see its 2D version in Matsakos et al. (2013). 


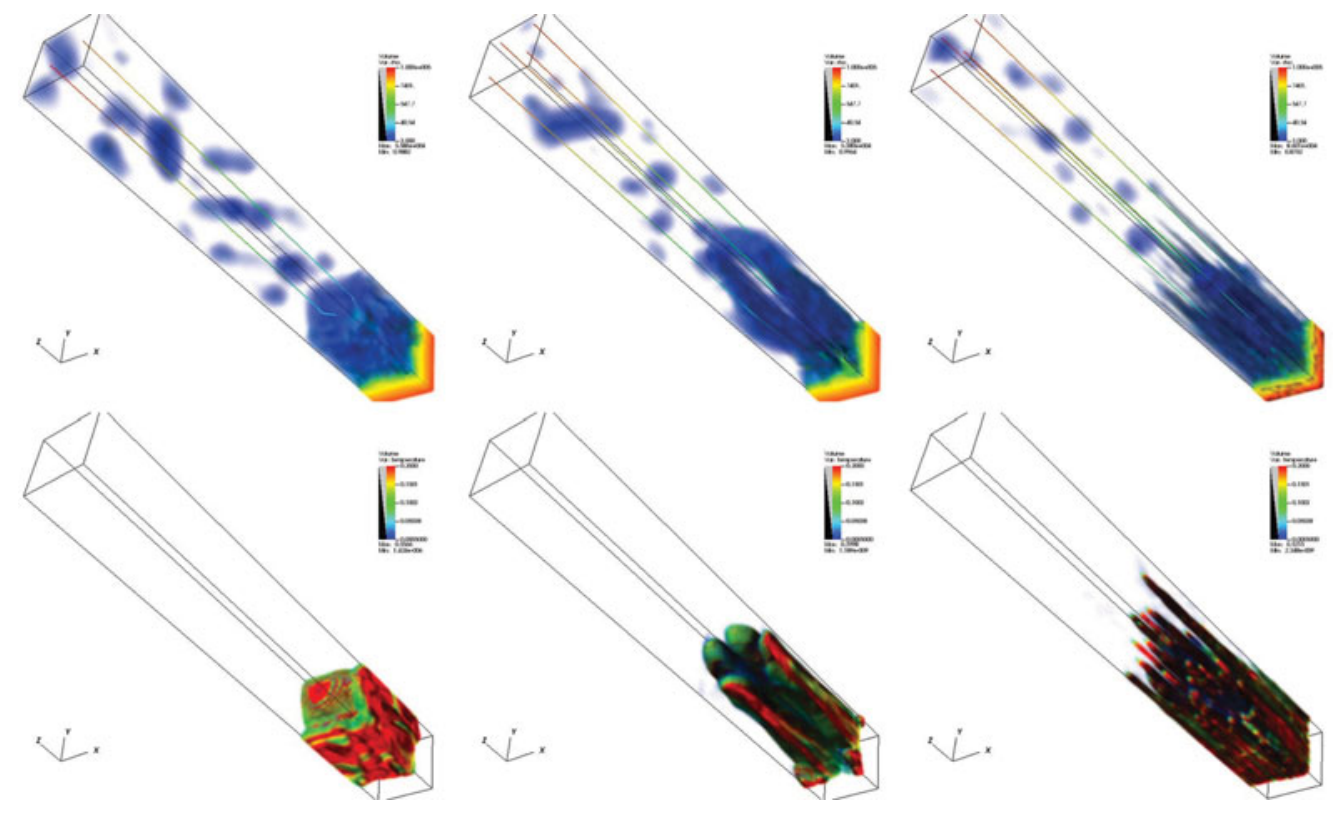

Figure 2. Volume plots of the density (top panels) and the temperature (bottom panels, only the million Kelvin regions are shown). From left to right, the magnetic field is assumed 30 G, $100 \mathrm{G}$, and $300 \mathrm{G}$. In each plot we can identify three regions: the clumpy stream, the hot post-shock region, and the chromosphere.

\section{Results}

In the case of a weak magnetic field (left panels of Fig. 2), the thermal pressure in the post-shock region dominates the magnetic (post-shock plasma- $\beta$ larger than unity). Therefore, the inhomogeneities that appear in the hot slab, due to the clumps and the chromospheric variability, are quickly smoothed out. This leads to plasma mixing and a post-shock that shows chaotic motion with local velocities less but close to the infalling gas speed. Nevertheless, despite this turbulent structure, the system is found to retain globally the periodic formation-collapse pattern of the reverse shock.

When the post-shock has a plasma- $\beta$ close to unity, the vertical magnetic flux tubes cannot be easily deformed and elongated structures form in the post-shock (middle panels of Fig. 2). This effect can be seen even more clearly in the case of a strong magnetic field (right panels of Fig. 2). Here, the plasma- $\beta$ of the hot slab is smaller than unity and hence the plasma is well confined within the flux tubes. In turn, the post-shock acquires a rich structure that consists of a collection of fibrils, each one of which follows the 1D quasi-periodic behavior. In the absence of perturbations, the fibrils oscillate in phase and the emission of the accretion shock would show a global periodic signature. However, the perturbations introduced in the system bring quickly the almost independent oscillators out of phase. Consequently, the overall emission is expected to be smooth, hiding the individual oscillations.

In order to visualize the structure of the whole post-shock region, Fig. 3 displays a reconstruction of a $2 \mathrm{D}$ vertical cut of an accretion stream of radius $5 \cdot 10^{4} \mathrm{~km}$. This is achieved by putting different simulation moments of the computational box side by side, since the fibrils are independent from each other. We have chosen to show only the model with a strong magnetic field because young stars have surface values on the order of $\sim \mathrm{kG}$ (e.g. Johns-Krull 2007). Evidently, even in the case of a uniform magnetic field, 

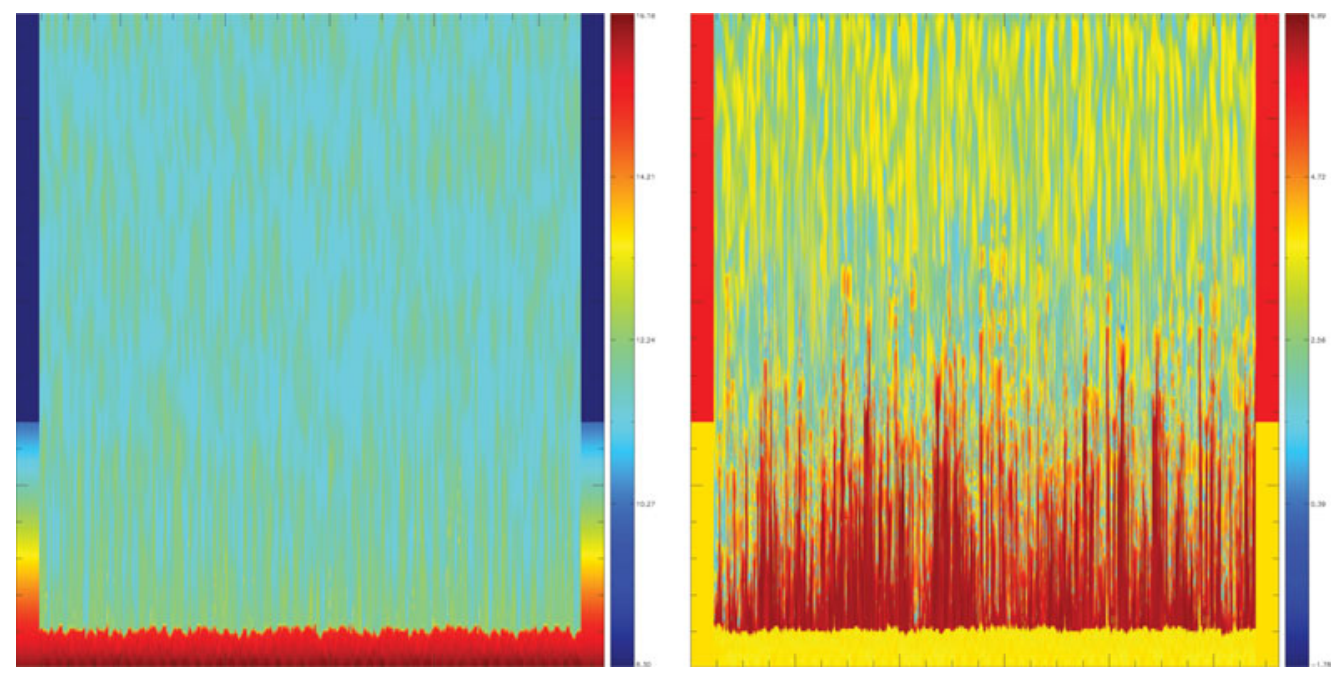

Figure 3. Density (left) and temperature (right) of the whole reconstructed accretion stream.

the presence of perturbations can create very rich structures in the post-shock region. Future work will focus on generating synthetic emission from such simulations in order to compare with observations.

\section{Acknowledgements}

This work was supported by the ANR STARSHOCK project (ANR-08-BLAN-0263072009/2013) and was granted access to the HPC resources of CINES under the allocation 2012-c2012046943 and 2013-c2013046943 made by GENCI (Grand Equipement National de Calcul Intensif).

\section{References}

Argiroffi, C., Maggio, A., \& Peres, G. 2005, A\& A, , 465, L5

Drake, J. J., Ratzlaff, P. W., Laming, J. M., \& Raymond, J., ApJ, , 703, 1224

Günther, H. M., Lewandowska, N., Hundertmark, M. P. G., et al. 2010, A\& A, , 518, 54

Koldoba, A. V., Ustyugova, G. V., Romanova, M. M., \& Lovelace, R. V. E. 2008, MNRAS, , 388,357

Johns-Krull, C. M. 2007, ApJ, , 664, 975

Matsakos, T., Chièze, J. P., Stehlé, C., et al. 2013, A\&A, , 557, 69

Orlando, S., Bonito, R., Argiroffi, C., et al. 2013, A\&A, , in press

Mignone, A., Bodo, G., Massaglia, S., et al. 2007, ApJS, , 170

Orlando, S., Sacco, G. G., Argiroffi, C., et al. 2010, A\&A, , 510, 71

de Sá, L. in preparation

Sacco, G. G., Argiroffi, C., Orlando, S., et al. 2008, A\&\&A, , 491, L17

Sacco, G. G., Orlando, S., Argiroffi, C., et al. 2010, $A \& A$, , 522, 55 